\title{
Eco-photonics:
}

\section{Micro-encapsulated probe as implantable sensor for monitoring the physiological state of water organisms}

\author{
A. Popov ${ }^{1,2}$, A. Gurkov ${ }^{3}$, E. Borvinskaya ${ }^{3,4}$, A. Sadovoy ${ }^{5}$, A. Bykov ${ }^{1,2}$, M. Timofeyev ${ }^{3}$, and I. Meglinski*,1,2,3,6 \\ ${ }^{1}$ University of Oulu, Optoelectronics and Measurement Research Unit, P.O. Box 4500, Oulu, 90014, Finland \\ ${ }^{2}$ ITMO University, 49 Konverkski pr., St. Petersburg, 197101, Russia \\ ${ }^{3}$ Institute of Biology, Irkutsk State University, 3 Lenina str., Irkutsk, 664003,Russia \\ ${ }^{4}$ Institute of Biology at Karelian Research Centre of Russian Academy of Sciences, Petrozavodsk, Russia \\ ${ }^{5}$ Institute of Materials Research and Engineering, A*STAR, 138634, Singapore \\ ${ }^{6}$ National Research Nuclear University MEPhI, Institute of Engineering Physics for Biomedicine (PhysBio), \\ Kashirskoe shosse, 31, 115409 Moscow, Russia \\ E-mail: igor.meglinski@oulu.fi
}

\begin{abstract}
Nowadays there is a growing interest to the natural evolutionary changes and especially those driven by environmental pollution and climatic variations. Climate change in combination with human activities largely influences the environment and especially aquatic ecosystems. We develop an approach for non-invasive screening of stress felt by water organisms due to environmental variations. In particular, we offer real-time quantitative assessment of internal temperature and $\mathrm{pH}$ in small aquatic species, such as shrimps, fish and fish embryos. The approach is based on the measurements of fluorescent and luminescent spectra obtained, respectively, from microencapsulated fluorescent dyes and upconversion particles embedded into the aquatic animals in vivo.
\end{abstract}

Keywords-micro-encapsulated probes, sensors, upconversion particles, fluorescence, luminescence, eco-photonics, aquatic organisms, environmental monitoring, climate change

\section{INTRODUCTION}

Nowadays there is a growing interest to the natural evolutionary changes and especially those that driven by environmental pollution and climate change. Climate change in combination with human activities largely influences the environment and especially the aquatic ecosystems. We develop an approach for non-invasive screening of stress felt by water organisms due to environmental variations. As an example we present the results of real-time quantitative assessment of internal temperature and $\mathrm{pH}$ in aquatic species.

\section{EXPERIMENTAL APPROACH}

Laser light was used to excite encapsulated SNARF-1-D with sequential emission signal acquisition in the green channel (587 $\mathrm{nm})$ and the red channel $(627 \mathrm{~nm})$ for further ratiometric $\mathrm{pH}$ measurements. Images of micro-encapsulated biomarkers MBMs (probes) in buffers and inside animals consist of three channels: a 587-nm channel, a 627-nm channel and a white light channel $[1,2]$. In a similar manner to $[1,2]$ luminescent signal from upconversion [Y2O3: $\mathrm{Yb}, \mathrm{Er}$ ] particles UCPs [3] was excited by a semiconductor laser ITC $4005(\lambda=975 \mathrm{~nm})$. The luminescence spectra were measured from the surface of the biological sample through an objective, a dichroic mirror, and

The authors would like to acknowledge support from the Academy of Finland (project Nos. 260321, 290596), EDUFI Fellowship (TM-17-10389), MEPhI Academic Excellence Project (Contract No. 02.a03.21.0005) and the Russian Science Foundation (grant No. 15-14-10008). optical fiber; photoluminescence signals were recorded by the spectrometer with an acquisition time $200 \mathrm{~ms}$.

\section{RESULTS}

The obtained images of fluorescent and luminescent signals produced, correspondingly, by MBMs and UCPs injected to the Zebrafish and shrimp, respectively, are shown in Figure 1.
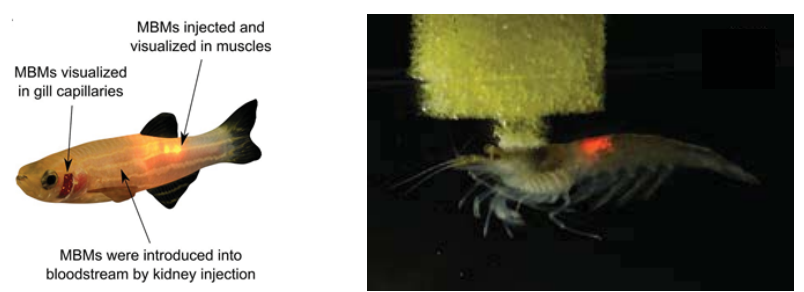

Fig.1. Zebrafish showing specific places where $\mathrm{pH}$ was measured with fluorescent MBMs (left); Image of C. multidentata shrimp with injected UCPs in vivo after illumination with laser light (right).

\section{SUMMARY}

The developed technique can be adopted for studies of various biosystems from terrestrial and aquatic invertebrates to fish and fish embryos. The proposed approach has strong potential to simultaneously measure a range of physiological characteristics using a set of micro-encapsulated probes and to finally bring toxicological bioassays and related research fields to a new level of effectiveness and sensitivity.

\section{REFERENCES}

[1] A. Gurkov, A. Sadovoy, E. Shchapova, C. Teh, I. Meglinski, and M. Timofeyev, "Microencapsulated fluorescent $\mathrm{pH}$ probe as implantable sensor for monitoring the physiological state of fish embryos", PLOS One, Vol.12, No.10, e0186548, 2017.

[2] E. Borvinskaya, A. Gurkov, E. Shchapova, S. Sadovoy, B. Baduev, I Meglinski and M. Timofeyev, "Parallel in vivo monitoring of $\mathrm{pH}$ in gill capillaries and muscles of fishes using microencapsulated biomarkers", Biology Open, Vol.6, No.5, pp.673 - 677, 2017.

[3] E. Volkova, I. Yanina, A. Popov, A. Bykov, A. Gurkov, E. Borvinskaya, M. Timofeev, and I. Meglinski, "Eco-photonics: assessment of temperature gradient in aquatic organisms by using upconversion luminescent particles”, Quantum Electron., Vol.47, pp.153 - 157, 2017. 